\title{
IKK-2 inhibitor TPCA-1 represses nasal epithe- lial inflammation in vitro
}

\author{
F. Sachse ${ }^{1}$, K. Becker ${ }^{2}$, T. Basel ${ }^{1}$, D. Weiss ${ }^{1}$, C. Rudack ${ }^{1}$ \\ Department of Otorhinolaryngology Head and Neck Surgery, University Hospital Münster, Germany \\ Institute of Medical Microbiology, University Hospital Münster, Germany
} SUMMARY

\begin{abstract}
Background: Nasal polyposis (NP) is considered a subgroup within chronic rhinosinusitis. $N P$ can be further subdivided into aspirin sensitive- and aspirin tolerant types (ASNP/ ATNP). Although the true etiology of NP has not been identified so far, it is agreed that $N P$ represents an inflammatory disease of the nasal mucosa. Alterations of cellular kinase activities including that of IKK-2 might play a role in this inflammatory process.

Methods: Paraffin sections of ASNP, ATNP and controls were immunostained with Phospho-IКB- $\alpha$ antibody that detects the direct IKK-2 product ( $I \kappa B-\alpha$. Intensity of epithelial staining was analysed semi-quantitatively by two independent observers. In cultured nasal polyp epithelial cells (NPECS) epithelial derived cytokines IL-8 and GRO- $\alpha$ were induced by TNF- $\alpha$ or Staphylococcal supernatants and subsequently repressed by IKK-2 inhibitor TPCA-1.

Results: Significant Phospho-I $\mathrm{\kappa} B-\alpha$ staining was observed in the nasal epithelium of ASNP compared to ATNP and controls suggesting strong IKK-2 activation in patients with ASNP in vivo. In vitro, pro-inflammatory cytokines $I L-8$ and GRO- $\alpha$ in NPECS were significantly repressed by TPCA-1.

Conclusion: IKK-2 activity is increased in the subgroup of ASNP. IL-8 and GRO- $\alpha$ responses were repressed by IKK-2 inhibitor TPCA-1 in vitro. IKK-2 inhibitors might represent a potential target for anti-inflammatory intervention in ASNP.
\end{abstract}

Key words: Chronic rhinosinusitis, nasal polyposis, IKK-2, NF-кB, Staphylococcus aureus

\section{INTRODUCTION}

Chronic rhinosinusitis (CRS) is an inflammatory disease of the nasal and paranasal mucosa of yet unknown etiology. CRS with nasal polyps (nasal polyposis, NP) is considered as a subgroup of CRS ${ }^{(1)}$. Glucocorticosteroids are still the drugs of first choice if medical treatment is intended. However, as oral steroids cannot be given over a longer period of time because of the well-known side effects, topical treatment with nasal steroids characterized by a minimum of systemic bioavailability has become the preferred modality of treatment. Nevertheless, even a combined treatment approach consisting of removal of the diseased sinus mucosa by endoscopic sinus surgery and preand postoperative topical treatment with nasal steroids cannot prevent recurrence of nasal polyps particularly in patients suffering from NP, asthma and aspirin sensitivity. Aside from glucocorticosteroids, a variety of substances are currently evaluated for their efficacy in the treatment of CRS but there is still need for novel anti-inflammatory drugs ${ }^{(2)}$.

The airway epithelium is considered to play an important role in CRS via the production of typical epithelial derived cytokines such as IL-8, Gro- $\alpha$, RANTES, MCP-1 which in turn promote chemotaxis of neutrophils, eosinophils and other type of leucocytes. In this process, nuclear factor kappa beta (NF-кB) is a major regulator of inflammatory genes that control the production of cytokines, chemokines and numerous pro-inflammatory mediators. NF- $\mathrm{kB}$ consists of two heterodimers of p50 (NF$\kappa \mathrm{B} 1)$ and p65 (RelA) that are bound to an IкB inhibitory protein that holds the complex in the cytoplasm. Upon appropriate stimulation of the cell, the IкB protein is phosphorylated and ubiquinated, which leads to subsequent proteasome-mediated

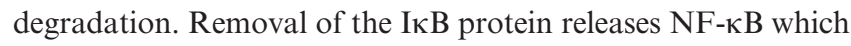
then translocates from the cytoplasm to the nucleus where it can initialize transcription of inflammatory genes (Figure 1). In the classical pathway, the critical phosphorylation of the IкB protein is mediated by the IкB kinase (IKK) complex, which consists of two catalytic (IKK-1 or IKK- $\alpha$ and IKK-2 or IKK$\beta$ ) and one regulatory subunit (IKK- $\gamma$, NEMO) ${ }^{(3-5)}$. IKK-2 has been shown to be 20 -fold more active than IKK-1 in the phosphorylation of $\mathrm{I} \kappa \mathrm{B}{ }^{(6)}$. Moreover, it has been shown that 
$\mathrm{NF}-\kappa \mathrm{B}$ activation in vivo solely depends on IKK-2, but not on IKK-1 ${ }^{(7-9)}$. These observations led to the development of selective IKK-2 inhibitors to test their potential in the treatment of inflammatory airway diseases. Birrell and coworkers inhibited IKK-2 using the IKK-2 selective inhibitor TPCA-1 in cultured primary human airway smooth muscle cells and demonstrated that TPCA-1 inhibited release of several cytokines. In addition, in a rodent model of asthma it has been shown that TPCA-1 was able to reduce airway eosinophilia ${ }^{(10,11)}$. These results are very interesting in the context of epithelial inflammation in NP since eosinophilia and upregulation of a variety of cytokines have been frequently observed in NP ${ }^{(12)}$.

We therefore characterised IKK-2 activity in the epithelium of NP patients by immunohistochemistry. In cultured nasal polyp epithelial cells (NPECs) epithelial derived cytokines were induced by TNF- $\alpha$ or Staphylococcal supernatants and subsequently repressed by IKK-2 inhibitor TPCA-1. TNF- $\alpha$ was chosen as a stimulus in the in vitro studies since it is a wellknown activator of IKK-2 and therefore can be considered as a reference. Staphylococcal supernatants of strain Newman (Enterotoxin A positive) were included because of the potential impact of $S$. aureus on nasal polyp pathogenesis.

\section{PATIENTS AND METHODS}

\section{Patients}

Nasal polyposis was diagnosed according to the criteria defined by the EPOS guidelines ${ }^{(1)}$. Due to the presence of aspirin intolerance, polyps were further subdivided into aspirin tolerant (ATNP / $\mathrm{n}=17$, males: 8 / females: 9; mean age: 46 years \pm 17.1(SD)) and aspirin sensitive nasal polyps (ASNP / $\mathrm{n}=12$, males: 8 / females: 4 ; mean age 54 years $\pm 16.5(\mathrm{SD})$ ). Inferior turbinate mucosa ( $\mathrm{TM} / \mathrm{n}=16$, males: $10 /$ females: 6; mean age: 31.2 years $\pm 14.1(\mathrm{SD})$ ), which was used as control tissue, was obtained from patients undergoing septoplasty or septorhinoplasty who additionally needed a reduction of the volume of their inferior turbinates. These patients did not suffer from CRS/ NP or any other type of nasal inflammation.

None of the patients underwent sinus surgery or turbinate surgery previously. In addition, none of the patients received medical treatment four weeks prior to surgery. Allergy was evaluated by allergic history and skin-prick test. In case of ambiguous results, specific IgE detection and/ or nasal provocation test were additionally performed. A diagnosis of asthma (ASNP 12/12, ATNP 5/17, TM 0/16) was verified by a pulmonary specialist. Aspirin sensitivity was evaluated by typical medical history and positive nasal provocation test.

All mucosal tissue samples were obtained during routine surgery at the Department of Otorhinolaryngology of the University Hospital of Münster, Germany. Informed consent was obtained from all patients and the study was approved by the ethics committee of the University of Münster.

\section{Nasal polyp epithelial cell culture}

Unless declared otherwise, all reagents were purchased from Sigma (Deisenhofen, Germany).
For establishment of the NPEC culture model nasal polyp tissue was obtained from two ASNP patients and dissected for individual experiments under sterile conditions. Subsequently, tissue was washed with phosphate-buffered saline (PBS) and incubated with trypsin $(0.5 \%)$ overnight at $4^{\circ} \mathrm{C}$. The epithelial layer was resuspended in PBS (pH 7.4). Following centrifugation at 800 RPM for 10 minutes, cells were washed again with PBS, pelleted and resuspended in a serum-free AECG medium (Airway epithelial cell growth medium, AECG Medium, Promocell, Heidelberg, Germany), supplemented with a readyto-use supplement mix according to the manufacturer's recommendation. Penicillin was applied at a dilution of 200 units/ $\mathrm{ml}$ medium and streptomycin applied to a dilution of $0.2 \mu \mathrm{g} /$ $\mathrm{ml}$ medium (Biochem, Berlin, Germany). NPECs were grown to $80 \%$ confluence and passaged two more times. NPECs were then split and grown in 12 well plates (each well containing about $1,8 \times 10^{5}$ cells for a single experiment) to $80 \%$ confluence. The medium was exchanged by serum-free medium 24 hours prior to stimulation.

The epithelial phenotype of cells was confirmed by staining of epithelial cells with a monoclonal anti-pan cytokeratin antibody as previously shown ${ }^{(13)}$. Viability of NPECs as assessed by trypan blue dye exclusion was greater than $95 \%$ in all experiments before and after stimulation.

Prior to stimulation with TNF- $\alpha, S$. aureus Newman and TPCA-1 time- and dose response curves were experimentally determined (results not shown). Based on these results, NPECs were incubated with prednisolone $\left(10^{-5} \mathrm{M}\right)$ or different concentrations of TPCA-1 $\left(10^{-7.5} \mathrm{M}, 10^{-6} \mathrm{M}\right.$ and $\left.10^{-5} \mathrm{M}\right)$. NPECs were stimulated with TNF- $\alpha(20 \mathrm{ng} / \mathrm{ml})$ or staphylococcal supernatants derived from the $S$. aureus strain Newman D2C (ATCC 2590, dilution 1:10) which has been tested positive for $S$. aureus enterotoxin A gene (SEA) ${ }^{(14,15)}$. Six independent experiments were performed for each stimulation/ inhibition experiment. Unstimulated controls were always included.

After 24 hours culture supernatants were collected and synthesis of IL-8 (detection range $>3.5 \mathrm{pg} / \mathrm{ml}$, R\&D, Wiesbaden, Germany) and GRO- $\alpha$ (detection range $>10 \mathrm{pg} / \mathrm{ml}, \mathrm{R} \& \mathrm{D}$, Wiesbaden, Germany) was quantified by ELISA according to the manufacturer's instructions.

Cytokine concentrations were expressed as means \pm S.E.M. Statistical significance was determined using one-way analysis of variance with a Bonferroni post-test. Significance was taken where $\mathrm{p}<0.05(*)$.

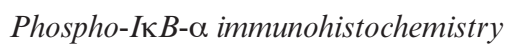

Since it is impossible to quantify kinase activity in different histological compartments in paraffin sections, we indirectly quantified IKK-2 activity by measuring the direct product of IKK-2 which is Phospho-IкB- $\alpha$. In airway epithelial cells it has been demonstrated that activation of NF- $\mathrm{KB}$ is dependent on IKK-2 which phosphorylates serines 32 and 36 of IкB- $\alpha$ before I $\mathrm{B}-\alpha$ degradation and final activation of NF- $\mathrm{KB}^{(16)}$. For immunohistochemical visualization of IкB- $\alpha$ in the nasal epithelium, we used the Phospho-IкB- $\alpha$ monoclonal antibody (Ser32/36, 5A5, Cell Signaling Technologies, Danvers, MA, USA) that detects 
IкB- $\alpha$ only when phosphorylated at serines $32 / 36$.

Immediately after surgery mucosal specimens were embedded in paraffin and $5 \mu \mathrm{m}$ thick sections were prepared. Sections were then deparaffinized and hydrated using xylene and graded concentrations of ethanol. Washing was performed twice with $\mathrm{dH}_{2} 0$ for 5 minutes. For unmasking of antigen, sections were heated in $10 \mathrm{mM}$ sodium citrate buffer ( $\mathrm{pH}$ 6.0) in a steamer at $90^{\circ} \mathrm{C}$. Slides were then cooled for 20 minutes and subsequently washed twice with $\mathrm{dH}_{2} 0$ for 5 minutes. Blocking of the endogenous peroxidase was perfomed by addition of $1 \%$ hydrogen peroxide for 10 minutes. Sections were then washed several times with $\mathrm{dH}_{2} 0$ and PBS. Sections were then incubated with the primary antibody Phospho-IкB- $\alpha$ at a dilution of 1:100 in PBS overnight at $4^{\circ} \mathrm{C}$. After that, sections were washed with PBS. For visualization the DAKO-LSAB-Kit (K0679, DAKO, Denmark) was used. Sections were then washed with $\mathrm{dH}_{2} 0$, counterstained with in Mayer's haematoxylin or 20s, blued in tap water and cover-slipped with Kaiser's glycerol gelatine. Isotype controls (Isotype Mouse IgG1 mAb, clone NCM1, Abcam) were always included and did not demonstrate aspecific staining.

In each specimen, five representative high-power-fields at 400x magnification were analysed for staining intensity by two independent investigators. Staining intensity was graded in relation to maximal $(100 \%)$ staining of the epithelium as shown in Figure 2B. Moderate staining (Figure 2D) was defined as equal or less than $50 \%$ of maximal staining activity, weak staining (Figure 2C) as equal or less than 10\% of maximal staining activity and absent staining as no staining at all $(0 \%)$. A mean staining intensity was calculated for each specimen and finally group specific means were calculated and compared using unpaired Mann-Whitney Rank sum test where $\mathrm{p}<0.05$ was considered statistically significant (Figure 3).

\section{RESULTS}

\section{Immunhistochemistry}

Immunohistochemistry revealed strong epithelial PhosphoIкB- $\alpha$ staining in ASNP (Figure $2 \mathrm{~A} / \mathrm{B}$ ) obtained from patients with aspirin sensitivity and asthma compared with ATNP (Figure 2C). In addition, more intense staining was also observed in inflammatory cells in the submucosal stroma in ASNP compared with ATNP. Loss of submucosal glands is very common in nasal polyps and was a frequent finding in ATNP and ASNP. By contrast, submucosal glands in TM displayed moderate to strong staining (Figure 2D), whereas weak staining of the epithelium and inflammatory cells was observed in a majority of cases.

A significant increase of Phospho-IкB- $\alpha$ staining intensity in ASNP compared with ATNP and TM was observed (Figure 3).

\section{Repression of cytokines in NPEC cell culture}

A significant increase of IL-8 and GRO- $\alpha$ synthesis was observed following TNF- $\alpha$ stimulation. In general, IL- 8 and GRO- $\alpha$ responses induced by TNF- $\alpha$ were weaker compared with induction by staphylococcal supernatants of the Newman strain. An increased basal rate of GRO- $\alpha$ was observed,

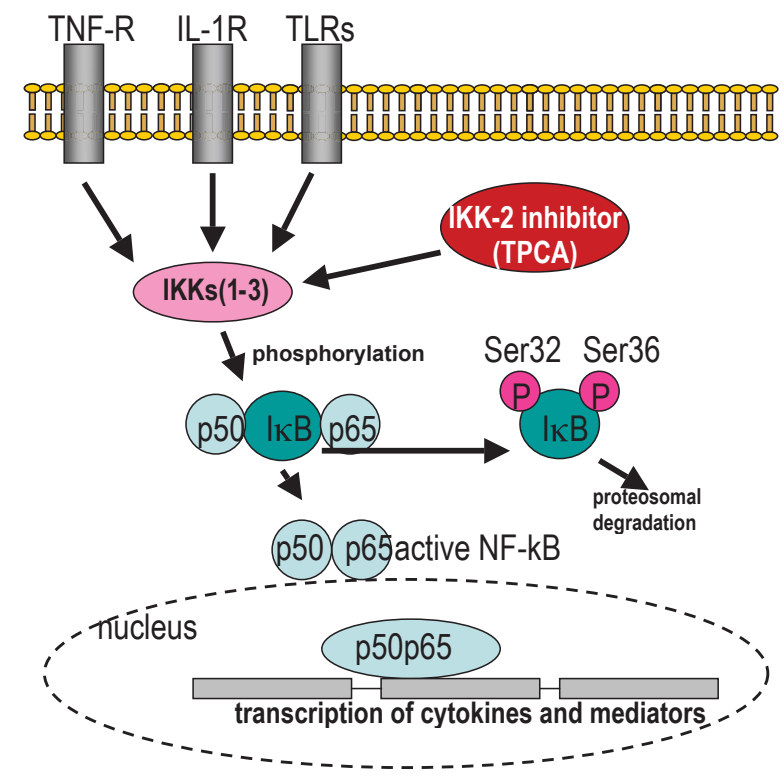

Figure 1. Simplified IKK pathway and activation of NF-kB. Activation of numerous receptors including the TNF-receptor (TNF$\mathrm{R}$ ), toll-like receptors (TLRs) or the IL-1 receptor (IL-1R) result in IKK-mediated phosphorylation of the IkB protein that is then ubiquinated and subsequently undergoes proteasome-mediated degradation. Removal of the IkB protein releases NF-kB, which then translocates from the cytoplasm to the nucleus where it can initialize transcription of inflammatory genes.

whereas IL-8 was considerably induced after stimulation only (Figure 4/5).

TNF- $\alpha$ induced IL- 8 and GRO- $\alpha$ responses were significantly repressed by $10^{-5} \mathrm{M}$ prednisolone. TNF- $\alpha$ induced IL- 8 synthesis was significantly repressed by TPCA-1 at concentrations of $10^{-6} \mathrm{M}$ and $10^{-5} \mathrm{M}$, whereas GRO- $\alpha$ synthesis showed a decreasing tendency, but was not significantly repressed (Figure 4/5).

Experiments with Newman supernatants revealed significant induction of IL-8 and GRO- $\alpha$ synthesis after 24 hours. Preincubation with prednisolone at concentration of $10^{-5} \mathrm{M}$ resulted in a significant repression of IL-8 but not of GRO- $\alpha$ synthesis. TPCA- 1 at concentrations of $10^{-5} \mathrm{M}$ significantly repressed IL-8 and GRO- $\alpha$ synthesis after 24 hours (Figure 4/5).

\section{DISCUSSION}

The airway epithelium plays key role in the pathogenesis and disease exacerbation of chronic inflammatory diseases such as asthma, chronic obstructive pulmonary disease and CRS/ NP by the induction of numerous pro-inflammatory mediators. Since gene expression of pro-inflammatory mediators often involves the activation of the NF- $\mathrm{KB}$, this signaling pathway represents an interesting target for anti-inflammatory intervention. Signal-induced phosphorylation of inhibitory ІкB proteins by I $\mathrm{I} B$ kinases (IKK) is a critical step in the activation of NF- $\kappa$ B. Loss of I $\kappa$ B- $\alpha$ releases NF- $\kappa$ B which then translocates to the nucleus where it participates in the transcription of proinflammatory genes. Since it has been shown that IKK-2 is the principal kinase responsible for phosphorylation of serine resi- 


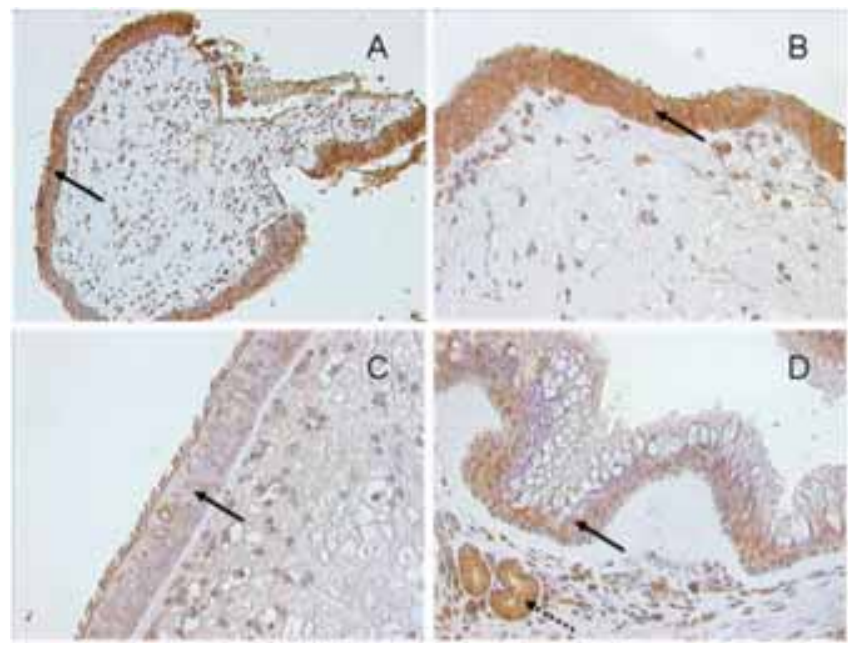

Figure 2. Phospho-IkB- $\alpha$ immunohistochemistry. Strong epithelial staining was observed in ASNP (A/B), whereas weak or moderate Phospho-IkB- $\alpha$ staining was observed in the epithelium of ATNP (C) and TM (D). Arrows indicate epithelial staining. The dotted arrow in (D) indicates strong staining of submucosal glands that are often lost in nasal polyps. Figures demonstrate representative staining results.

dues 32 and 36 of IкB- $\alpha$, selective inhibitors of this kinase were developed ${ }^{(17)}$. Recent studies indicate that IKK-2 inhibitors and glucocorticosteroids probably do not share a common mechanism of action and that selective inhibitors of IKK-2 may prove beneficial in situations where traditional corticosteroids have no effect ${ }^{(11,18)}$. This situation often is found in patients with NP particularly in those NP patients with asthma and aspirin sensitivity. Despite a combined treatment approach consisting of removal of the diseased sinus mucosa by endoscopic sinus surgery and pre- and postoperative topical treatment with nasal steroids recurrence of nasal polyps is frequently observed.

In this study, we have demonstrated strong epithelial PhosphoIкB- $\alpha$ staining in the subgroup of ASNP obtained from patients demonstrating the typical Samter's triad of nasal polyps, aspirin sensitivity and asthma (Figure $2 \mathrm{~A} / \mathrm{B}$ ). As described in the methods' section Phospho-IкB- $\alpha$ staining can be used as an indirect marker of IKK-2 activity in airway epithelial cells. According to the results of immunohistochemistry, we hypothesize that IKK-2 activity is increased in ASNP, thus leading to activation of NF- $\kappa \mathrm{B}$ and finally increased synthesis of pro-inflammatory mediators including numerous cytokines. Such a mechanism is also corroborated by a study demonstrating significant correlations between the degree of epithelial NF- $\mathrm{BB}$ activation and the levels of IL-8, IL-16, and eotaxin mRNA expression ${ }^{(19)}$. Due to the selection of the patients in this study, we cannot exclude the possibility that, apart from aspirin sensitivity, asthma alone or as a contributing factor was responsible for the increase of IKK-2 activity since both factors were observed in ASNP. Therefore, it is impossible to clearly define the impact of aspirin sensitivity and/or asthma on IKK-2 activity in ASNP. In ATNP, however, asthma was present in five cases and here only moderate Phospho-IкB- $\alpha$ staining was observed (Figure 2C).

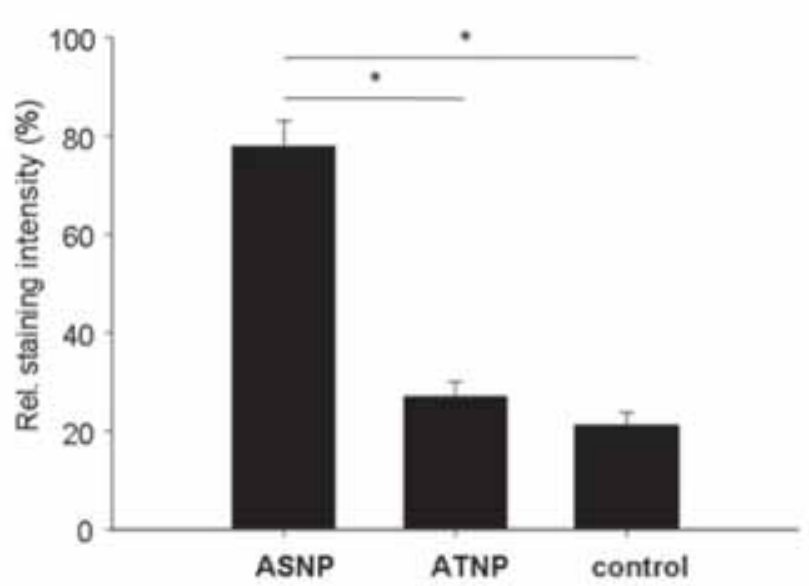

Figure 3. Staining intensity of Phospho-IkB- $\alpha$ immunohistochemistry. Columns represent means \pm S.E.M of staining intensity of ASNP (n $=12)$, ATNP $(\mathrm{n}=17)$ and TM $(\mathrm{n}=16) . \mathrm{P}<0.05(*)$ was considered statistically significant.

As immunohistochemistry revealed Phospho-IкB- $\alpha$ staining in the epithelium, we consequently used nasal epithelial cells for in vitro experiments and quantified typical epithelial cytokines such as IL 8 and GRO- $\alpha$ to study the effects of IKK-2 inhibitor TPCA-1 in a model of nasal epithelial inflammation. In nasal epithelial cells obtained from ASNP we could demonstrate that epithelial IL-8 synthesis was significantly repressed by TPCA-1. Moreover, repression by TPCA-1 was at least as effective as by the glucocorticosteroid prednisolone.

Birrell and coworkers demonstrated IKK-2 inhibition both in lipopolysaccharide and allergen-sensitized in vivo models of lung inflammation ${ }^{(10,11)}$. These results indicate that IKK-2 inhibition is not restricted to a single specific pro-inflammatory stimulus. In NP numerous studies aimed to identify pathogens as a pro-inflammatory trigger including virus, fungi and bacteria, but results are still controversial. As some features observed in nasal polyp pathology can be explained by $S$. aureus enterotoxins (SEA), we also included $S E A$-positive staphylococcal supernatants derived from $S$. aureus strain Newman as a pro-inflammatory stimulus in the cell culture experiments. We found that staphylococcal supernatants driven increase of IL-8 and GRO- $\alpha$ synthesis was significantly repressed by TPCA-1.

Our results are also interesting in another context. Oral and nasal administration of aspirin is used to treat aspirin sensitive patients with nasal polyps. In fact, several studies have documented benefit of aspirin desensitization in patients with ASPN by improving symptoms of rhinosinusitis, reduction of systemic corticosteroids and a diminished recurrence rate of nasal polyps ${ }^{(20-22)}$. Although the pathomechanism of aspirin induced asthma and nasal polyps still is not fully understood, evidence exists that intolerance to aspirin is not an IgE-mediated allergic reaction but it associated with abnormal metabolism of arachidonic 

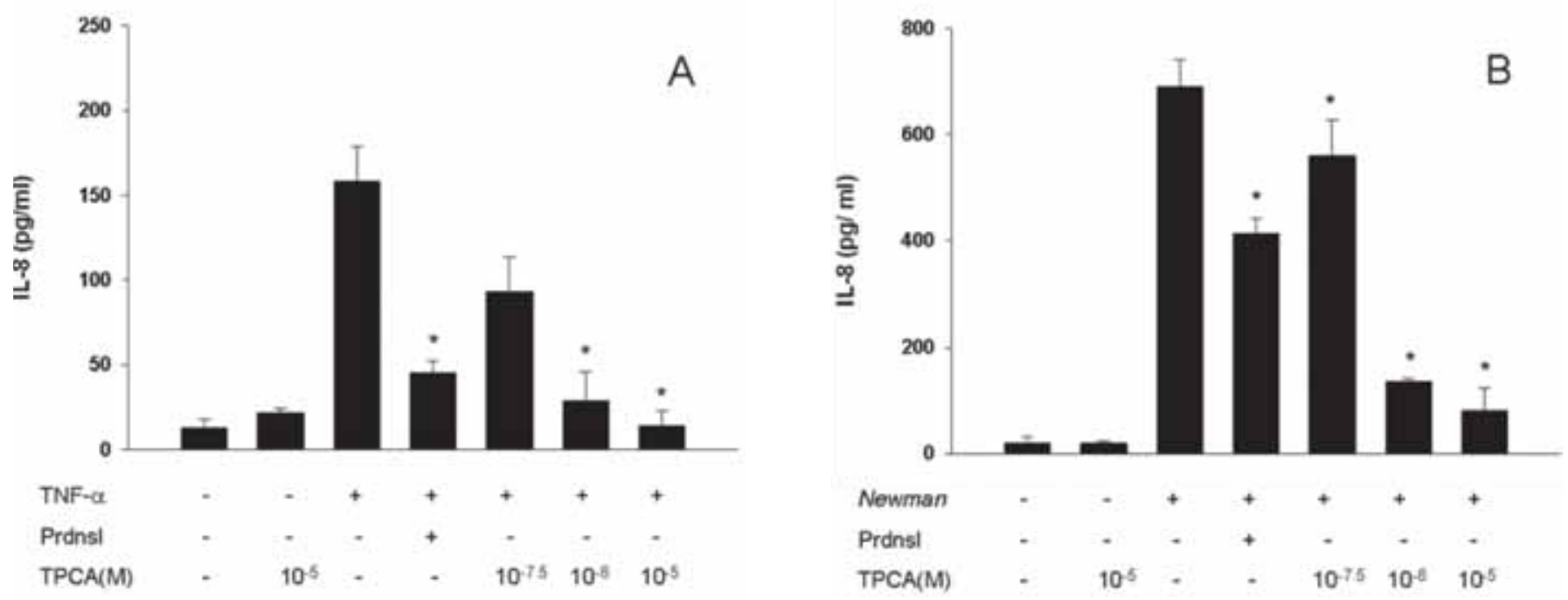

Figure 4 A/B. IL-8 and synthesis by NPECs and repression by TPCA-1. TNF- $\alpha$ was added at a concentration of $20 \mathrm{ng} / \mathrm{ml}$. Prednisolone (Prdnsl) was added at a concentration of $10^{-5} \mathrm{M}$. Staphylococcal supernatants of strain Newman (Newman) were added at dilution of 1:10. Bars represent means \pm S.E.M. of six independent experiments. Figures in brackets indicate TPCA concentrations. P $<0.05\left(^{*}\right)$ was considered significant.
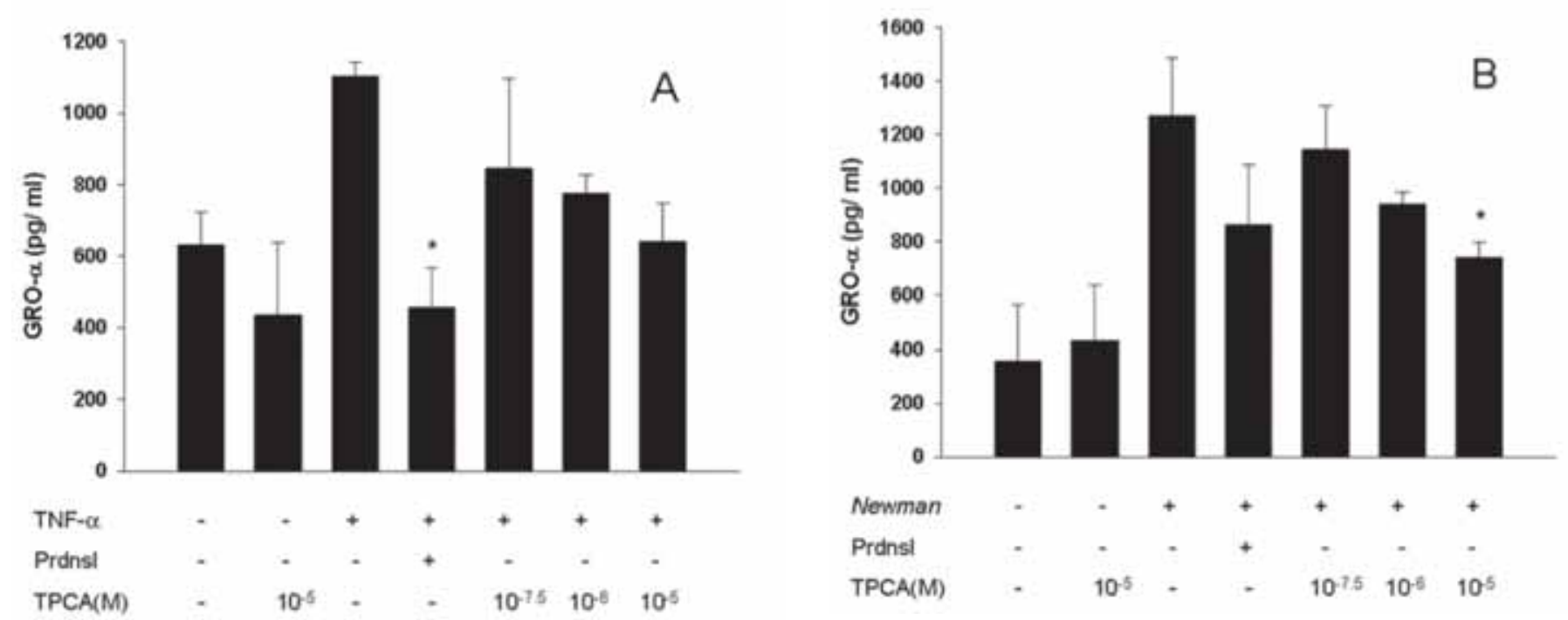

Figure 5 A/B. GRO-a synthesis in NPECs and repression by TPCA-1. TNF- $\alpha$ was added at a concentration of $20 \mathrm{ng} / \mathrm{ml}$. Prednisolone (Prdnsl) was added at a concentration of $10^{-5} \mathrm{M}$. Staphylococcal supernatants of strain Newman (Newman) were added at dilution of 1:10. Bars represent means \pm S.E.M. of six independent experiments. Figures in brackets indicate TPCA concentrations. $\mathrm{P}<0.05(*)$ was considered significant.

acids. Following aspirin challenge, arachidonic acid metabolites are diverted to the lipoxygenase pathway. This shift results in a decrease of anti-inflammatory prostaglandins and excessive production of pro-inflammatory leukotrienes that are responsible for the inflammatory reaction including severe asthma attacks observed in these aspirin sensitive patients ${ }^{(23)}$. Even though the exact mechanism of aspirin desensitization still is unknown, modulation of the synthesis of prostaglandins, leukotrienes and their receptors has been observed ${ }^{(24-26)}$.

Another possible mechanism of aspirin desensitization is intervention at the NF-кB pathway. Kopp and Gosh demonstrated inhibition of NF- $\kappa \mathrm{B}$ by sodium-salicylate and aspirin ${ }^{(27)}$. Frantz and O'Neill, however, commented that sodium-salicylate and aspirin rather inhibit cellular kinases and that NF- $\mathrm{KB}$ inhibition is not a unique effect of this anti-inflammatory agent ${ }^{(28)}$. Furthermore, Yin and coworkers demonstrated that aspirin and salicylate inhibits the activity of IKK-2 ${ }^{29)}$. This is very interesting in the context of the results presented in this study since we demonstrated repression of cytokines IL- 8 and GRO- $\alpha$ in NPECs obtained from ASNP by the selective IKK-2 inhibitor TPCA-1. It might be therefore hypothesized that at least some of anti-inflammatory effects observed in aspirin-sensitive patients with nasal polyps occur due to aspirin mediated inhibition of IKK-2 in the nasal epithelium. This might also explain why topical nasal administration of aspirin is successful in 
reducing the recurrence rate of nasal polyps as demonstrated by Patriarca et al. ${ }^{(30)}$.

Overall, using immunohistochemistry we demonstrated strong IкB- $\alpha$ staining, which served as an indirect marker of IKK-2 activity, in aspirin sensitive nasal polyps. Using a cell culture model of nasal epithelial inflammation IL-8 and GRO- $\alpha$ synthesis was significantly repressed by IKK-2 inhibitor TPCA-1. IKK-2 inhibitors therefore represent an interesting target for anti-inflammatory intervention in NP.

\section{AUTHOR CONTRIBUTIONS}

F.S. planned, performed and supervised all parts of the study. In addition, the manuscript was written by this person. K.B. prepared the staphylococcal supernatants used in the stimulation experiments. T.B. performed the immunohistochemistry in this study and evaluated the staining intensity. D.W. performed the cell culture experiments and inhibition studies with TPCA-1 C.R. supervised the study and considerably contributed in writing the manuscript.

\section{CONFLICT OF INTEREST}

The authors declare that there is no conflict of interest

\section{REFERENCE}

1. Thomas M, Yawn BP, Price D, Lund V, Mullol J, Fokkens W. EPOS Primary Care Guidelines: European Position Paper on the Primary Care Diagnosis and Management of Rhinosinusitis and Nasal Polyps 2007 - a summary. Prim Care Respir J. 2008; 17: 79-89.

2. Bachert C, Watelet JB, Gevaert P, van Cauwenberge P. Pharmacological management of nasal polyposis. Drugs. 2005; 65: 1537-1552.

3. Scheidereit C. Signal transduction. Docking IkappaB kinases. Nature. 1998; 395: 225-226.

4. Karin M. The beginning of the end: IkappaB kinase (IKK) and NF-kappaB activation. J Biol Chem. 1999; 274: 27339-27342.

5. Courtois G, Smahi A, Israel A. NEMO/IKK gamma: linking NF-kappa B to human disease. Trends Mol Med. 2001; 7: 427-430.

6. Mercurio F, Zhu H, Murray BW, et al. IKK-1 and IKK-2: cytokine-activated IkappaB kinases essential for NF-kappaB activation. Science. 1997; 278: 860-866.

7. $\mathrm{Hu} \mathrm{Y,} \mathrm{Baud} \mathrm{V,} \mathrm{Delhase} \mathrm{M,} \mathrm{et} \mathrm{al.} \mathrm{Abnormal} \mathrm{morphogenesis} \mathrm{but}$ intact IKK activation in mice lacking the IKKalpha subunit of IkappaB kinase. Science. 1999; 284: 316-320.

8. Shimada T, Kawai T, Takeda K, et al. IKK-i, a novel lipopolysaccharide-inducible kinase that is related to IkappaB kinases. Int Immunol. 1999; 11: 1357-1362.

9. Li Q, Lu Q, Hwang JY, et al. IKK1-deficient mice exhibit abnormal development of skin and skeleton. Genes Dev. 1999; 13: 13221328.

10. Birrell MA, Hardaker E, Wong S, et al. Ikappa-B kinase-2 inhibitor blocks inflammation in human airway smooth muscle and a rat model of asthma. Am J Respir Crit Care Med. 2005; 172: 962-971.

11. Birrell MA, Wong S, Hardaker EL, et al. IkappaB kinase-2-independent and -dependent inflammation in airway disease models: relevance of IKK-2 inhibition to the clinic. Mol Pharmacol. 2006; 69: 1791-1800.

12. Rudack C, Stoll W, Bachert C. Cytokines in nasal polyposis, acute and chronic sinusitis. Am J Rhinol. 1998; 12: 383-388.

13. Rudack C, Maune S, Eble J, Schroeder JM. The primary role in biologic activity of the neutrophil chemokines IL-8 and GROalpha in cultured nasal epithelial cells. J Interferon Cytokine Res. 2003; 23: 113-123.

14. Becker K, Roth R, Peters G. Rapid and specific detection of toxigenic Staphylococcus aureus: use of two multiplex PCR enzyme immunoassays for amplification and hybridization of staphylococcal enterotoxin genes, exfoliative toxin genes, and toxic shock syndrome toxin 1 gene. J Clin Microbiol. 1998; 36: 2548-2553.

15. Sachse F, von Eiff C, Stoll W, Becker K, Rudack C. Induction of CXC chemokines in A549 airway epithelial cells by trypsin and staphylococcal proteases - a possible route for neutrophilic inflammation in chronic rhinosinusitis. Clin Exp Immunol. 2006; 144: 534-542.

16. Catley MC, Chivers JE, Holden NS, Barnes PJ, Newton R. Validation of IKK beta as therapeutic target in airway inflammatory disease by adenoviral-mediated delivery of dominant-negative IKK beta to pulmonary epithelial cells. Br J Pharmacol. 2005; 145: 114-122.

17. Karin M, Yamamoto Y, Wang QM. The IKK NF-kappa B system: a treasure trove for drug development. Nat Rev Drug Discov. 2004; 3: $17-26$.

18. Newton R, Holden NS, Catley MC, et al. Repression of inflammatory gene expression in human pulmonary epithelial cells by smallmolecule IkappaB kinase inhibitors. J Pharmacol Exp Ther. 2007; 321: 734-742.

19. Takeno S, Hirakawa K, Ueda T, Furukido K, Osada R, Yajin K. Nuclear factor-kappa B activation in the nasal polyp epithelium: relationship to local cytokine gene expression. Laryngoscope. 2002; 112: 53-58.

20. Stevenson DD, Pleskow WW, Simon RA, et al. Aspirin-sensitive rhinosinusitis asthma: a double-blind crossover study of treatment with aspirin. J Allergy Clin Immunol. 1984; 73: 500-507.

21. Sweet JM, Stevenson DD, Simon RA, Mathison DA. Long-term effects of aspirin desensitization--treatment for aspirin-sensitive rhinosinusitis-asthma. J Allergy Clin Immunol. 1990; 85: 59-65.

22. Stevenson DD, Hankammer MA, Mathison DA, Christiansen SC, Simon RA. Aspirin desensitization treatment of aspirin-sensitive patients with rhinosinusitis-asthma: long-term outcomes. J Allergy Clin Immunol. 1996; 98: 751-758.

23. Szczeklik A, Sanak M, Nizankowska-Mogilnicka E, Kielbasa B. Aspirin intolerance and the cyclooxygenase-leukotriene pathways. Curr Opin Pulm Med. 2004; 10: 51-56.

24. Juergens UR, Christiansen SC, Stevenson DD, Zuraw BL. Inhibition of monocyte leukotriene B4 production after aspirin desensitization. J Allergy Clin Immunol. 1995; 96: 148-156.

25. Arm JP, Austen KF. Leukotriene receptors and aspirin sensitivity. N Engl J Med. 2002; 347: 1524-1526.

26. Parikh AA, Scadding GK. Intranasal lysine-aspirin in aspirin-sensitive nasal polyposis: a controlled trial. Laryngoscope. 2005; 115 : 1385-1390.

27. Kopp E, Ghosh S. Inhibition of NF-kappa B by sodium salicylate and aspirin. Science. 1994; 265: 956-959.

28. Frantz B, O'Neill EA. The effect of sodium salicylate and aspirin on NF-kappa B. Science. 1995; 270: 2017-2019.

29. Yin MJ, Yamamoto Y, Gaynor RB. The anti-inflammatory agents aspirin and salicylate inhibit the activity of I (kappa) B kinase-beta. Nature. 1998; 396: 77-80.

30. Patriarca G, Schiavino D, Nucera E, Papa G, Schinco G, Fais G. Prevention of relapse in nasal polyposis. Lancet. 1991; 337: 1488.

\author{
F. Sachse, MD \\ Department of Otorhinolaryngology \\ Head and Neck Surgery \\ University of Muenster \\ Kardinal von Galen Ring 10 \\ D-48149 Muenster \\ Germany
}

Tel: $\quad+49-251-83-56863$

Fax: +49-251-83-56812

E-mail: sachsef@ukmuenster.de 